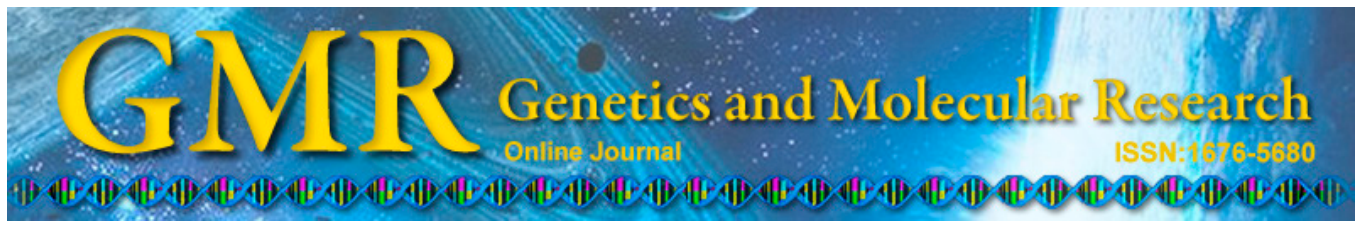

$\underline{\text { Case Report }}$

\title{
Visceral leishmaniasis diagnosed by biopsy of an axillary lymph node: case report and literature review
}

\section{Z.Z. Liu and X.J. Lv}

Center of Infectious Diseases, West China Hospital of Sichuan University, Chengdu, China

Corresponding author: X.J. Lv

E-mail: xiaojulv@126.com

Genet. Mol. Res. 13 (4): 9960-9963 (2014)

Received March 17, 2014

Accepted July 29, 2014

Published November 27, 2014

DOI http://dx.doi.org/10.4238/2014.November.27.25

\begin{abstract}
A 56-year-old man presented with fevers up to $40^{\circ} \mathrm{C}$ and acute pain in the left upper quadrant. Full blood count was normal and biochemical tests revealed increased serum globulin. Abdominal ultrasound revealed hepatomegaly and splenomegaly with multiple enlarged lymph nodes in the neck, axilla, and inguinal regions. The bone marrow smear was negative. Splenectomy was performed and the pathology revealed splenic ischemic infarcts. Biopsy of one of the axillary lymph nodes revealed macrophages filled with leishmanias. The patient tested positive by the rk39 dipstick test. He was treated with sodium stibogluconate and showed full recovery.
\end{abstract}

Key words: Kala-azar; Lymph node biopsy; rk39 dipstick 


\section{INTRODUCTION}

Leishmaniasis is an infectious disease caused by the protozoan Leishmania spp. Leishmaniasis is found throughout the world, except in Australia and the Antarctic. There are an estimated 500,000 new cases each year, with 41,000 recorded deaths in the year 2000 (Guerin et al., 2002). Three clinical forms of leishmaniasis have been identified, including visceral leishmaniasis (VL), cutaneous leishmaniasis, and mucocutaneous leishmaniasis. Patients with VL typically present with fever, abdominal pain, splenomegaly, hepatomegaly, cachexia, and pancytopenia. The parasites usually invade the spleen, bone marrow, lymph nodes, or liver. VL is widely distributed in the 6 provinces in the west of China, particularly in the XinJiang, GanSu, and SiChuan provinces, and shows a scattered distribution. VL patients are often misdiagnosed or experience missed diagnosis.

\section{CASE REPORT}

A 56-year-old male first presented with an acute onset of fevers of up to $40^{\circ} \mathrm{C}$ associated with pain in the left upper quadrant at a local hospital 2 years before admission. An abdominal ultrasound revealed cholecystolithiasis. He was given antibiotics and his symptoms resolved. Eighteen months ago, the pain relapsed and abdominal ultrasound showed splenomegaly and splenic abscess. Splenectomy was performed and pathology revealed multiple coagulation necrotizing loci. The surrounding spleen tissue was infiltrated by some lymphocytes and plasma cells. Immunohistochemistry stain results were as follows: lymphocytes in the splenic corpuscle were $\mathrm{CD} 20+$, peripheral cells were $\mathrm{CD} 3+$, scattered plasma cells were $\mathrm{CD} 138+$, and granulocytes were MPO+. Polymerase chain reaction detected non-clonal rearrangement. Congo red staining was negative. Bone marrow smears were negative for parasites. Two months ago, the patient experienced recurrent fevers associated with lethargy and dizziness. He was referred to our hospital. He was a resident of WangCang County, GuangYuan, a city of Sichuan Province, an endemic area for leishmaniasis. His past medical history was non-significant. He did not smoke cigarettes or drink alcohol, or consume illicit drugs. Physical examination was noted for multiple enlarged lymph nodes in the neck, axilla, and inguinal regions. The laboratory findings were: hemoglobin $13.4 \mathrm{~g} / \mathrm{dL}$, leukocyte count $8780 / \mathrm{mm}^{3}$, neutrophilic granulocyte $25 \%$, lymphocytes $59 \%$, and platelets $220,000 / \mathrm{mm}^{3}$. Liver function studies showed that alanine aminotransferase was $72 \mathrm{IU} / \mathrm{L}$, aspartate aminotransferase was $47 \mathrm{IU} / \mathrm{L}$, serum albumin was $33.7 \mathrm{~g} / \mathrm{L}$, and serum globin was $91.9 \mathrm{~g} / \mathrm{L}$. Serum protein electrophoresis and immunoelectrophoresis were normal. A human immunodeficiency virus-antibody enzyme-linked immunosorbent assay test was negative. Bone marrow smears showed no abnormalities. The biopsy of the left axillary lymph node showed that the lymph node architecture was normal and follicle proliferation was active (Figure 1A). Multiple small basophilic pathogens were detected in the microphages both inside and outside of the germinal centers. These manifestations were consistent with leishmania infection (Figure 1B and C). The recombinant k39 antigen (rk39) dipstick test was positive. The patient was diagnosed with VL. Treatment was initiated with intravenous sodium stibogluconate, $120 \mathrm{mg}$ daily for 7 days. The patient remained asymptomatic at follow-up 6 months later. 


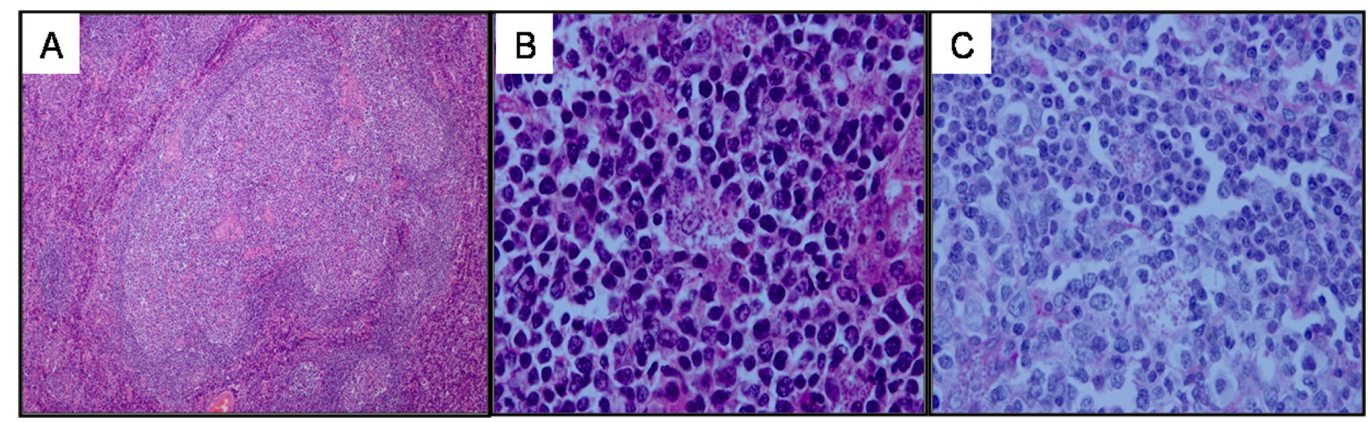

Figure 1. Histological photographs of lymph node. A. Hematoxylin and eosin staining (100X). B. Hematoxylin and eosin staining (1000X). C. Periodic Acid-Schiff stain (1000X).

\section{DISCUSSION}

$\mathrm{VL}$, which is also known as kala-azar, is a parasitic disease of the mononuclear phagocyte system. Leishmaniasis donovani is found in India, East Africa, and China. VL is transmitted by female sandflies of the genus Lutzomyia in the Americas and Phlebotomus elsewhere. Mammals such as rodents and canines are reservoir hosts of the infection and humans are incidental hosts. The major clinical manifestations of the disease include fever, splenomegaly in $90 \%$ of cases, and hepatomegaly in $75 \%$ of cases, which may be accompanied by a mild increase in serum aminotransferases and alkaline phosphatase, anemia, thrombocytopenia, and leucopenia (Bryceson, 1996; Pearson et al., 2000). Malaise, headache, dizziness, anorexia, and weight loss may also occur in the early stages, with darkening or even blackening of the skin (Pearson et al., 2000). This disease is classically diagnosed by the identification of parasites in the aspirates of the spleen, bone marrow, lymph nodes, or liver (Guerin et al., 2002). Delays in diagnosis are common, particularly in the early stage of the disease, because of non-specific symptomatology and difficulty in demonstrating intracellular protozoa in tissue aspirates (Cohen and Fields, 1988).

We describe a case of VL with intermittent fevers, acute pain in the left upper quadrant, increased serum globulin, hepatomegaly, multiple splenic infarcts, and multiple enlarged lymph nodes in the neck, axilla, and inguinal regions, without pancytopenia, as diagnosed by axillary lymph node biopsy. The absence of pancytopenia was atypical. The negative result of splenic pathology once and bone marrow smears twice were also rare. The protozoan is isolated in the bone marrow biopsy in $90 \%$ of cases (Pearson et al., 2000). Splenic aspiration has been reported to have a sensitivity of $98 \%$ for detecting VL (Guerin et al., 2002). Lymph node aspiration for diagnosing VL has not been thoroughly examined. When compared with splenic aspirates in the Sudan, 2 small studies found that lymph node aspiration (LNA) has low sensitivities of $56 \%(\mathrm{~N}=19)$ and $54 \%(\mathrm{~N}=87)$, respectively. In a series of 30 confirmed Sudanese VL cases, Leishmania parasites were found in 30 of 30 LNAs. In 2 cases, parasites were found in LNAs and bone marrow aspirates but not in splenic aspirates, and in 1 case they were found in LNAs and splenic aspirates, but not in bone marrow aspirates (Siddig et al., 1988; Zijlstra et al., 1992).

The rk39 is a 39-amino-acid protein, and has been cloned and expressed in Escherichia coli, from the $\mathrm{C}$ terminus of the kinesin protein of Leishmania major (Burns et al., 1993). This antigen has been reported to be highly specific for diagnosing kala-azar (Burns et 
al., 1993; Singh et al., 1995; Bern et al., 2000). The rk39 dipstick assay is more rapid, specific, sensitive, and less invasive than conventional methods used for diagnosing VL (Qu et al., 2000).

Pentavalent antimony is the preferred therapeutic agent for VL because of its effectiveness and cost, but resistance, complications, and adverse events have been reported. Treatment with liposomal amphotericin B is less toxic than pentavalent antimony and shows similar effectiveness.

\section{REFERENCES}

Bern C, Jha SN, Joshi AB, Thakur GD, et al. (2000). Use of recombinant K39 dipstick test and the direct agglutination test in a setting endemic for visceral leishmaniasis in Nepal. Am. J. Trop. Med. Hyg. 63: 153-157.

Bryceson ADM (1996). Leishmaniasis. In: Manson’s Tropical Diseases (Cook GC, ed.). WB Saunders, London, 12131245.

Burns JM JR, Shreffler WG, Benson DR, Ghalib HW, et al. (1993). Molecular characterization of a kinesin-related antigen of Leishmania chagasi that detects specific antibody in African and American visceral leishmaniasis. Proc. Natl. Acad. Sci. USA 90: 775-779.

Cohen D and Fields S (1988). CT findings in visceral leishmaniasis mimicking lymphoma. Comput. Med. Imaging Graph. 12: 325-327.

Guerin PJ, Olliaro P, Sundar S, Boelaert M, et al. (2002). Visceral leishmaniasis: current status of control, diagnosis, and treatment, and a proposed research and development agenda. Lancet lnfect. Dis. 2: 494-501.

Pearson R, De Queiroz Sousa A and Jeronimo S (2000). Leishmania species: visceral (kala-azar), cutaneous, and mucosal leishmaniasis. In: Mandell, Douglas and Bennett's Principles and Practice of Infectious Diseases (Mandell GL, Bennett JE and Dolin R, eds.). Churchill Livingston, Philadelphia, 2831-2845.

Qu JQ, Guan LR, Shulidan I, Zuo XP, et al. (2000). Rapid screening with a recombinant antigen (rK39) for diagnosis of visceral leishmaniasis using dipstick. Zhongguo Ji Sheng Chong Xue Yu Ji Sheng Chong Bing Za Zhi 18: 155-158.

Siddig M, Ghalib H, Shillington DC and Petersen EA (1988). Visceral leishmaniasis in the Sudan: comparative parasitological methods of diagnosis. Trans. R. Soc. Trop. Med. Hyg. 82: 66-68.

Singh S, Gilman-Sachs A, Chang KP and Reed SG (1995). Diagnostic and prognostic value of rK39 antigen in Indian leishmaniasis. J. Parasitol. 81: 1000-1003.

Zijlstra EE, Ali MS, El-Hassan AM, el-Toum IA, et al. (1992). Kala-azar: a comparative study of parasitological methods and the direct agglutination test in diagnosis. Trans. R. Soc. Trop. Med. Hyg. 86: 505-507. 